\title{
قواعد علم البيئة
}

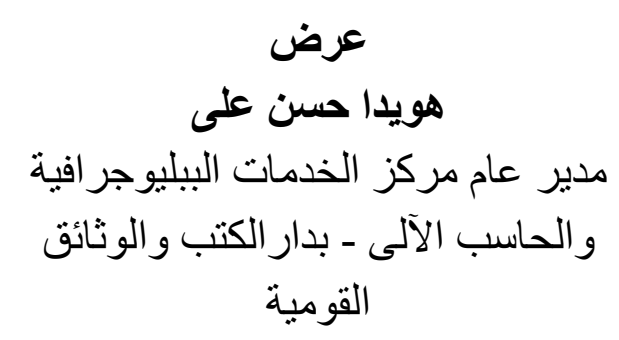

و الكوكبي قد حقق كثيرًا من النجاحات وكثبرًا من الإخفاقات. السبب في تللك الإخفاقات يعتقد أنها بسبب نقص الإحس المعرفة نحو البيئة و الإيكولوجي و الوظائف الطبيعية، وثبث أن اللجوء إلى تعليم البيئة هو العلاج. ظهور علم البيئة البوم كحقيقة قائمـة جعلنا نشعر بأهمية النظم البيئية التي تعبن التوم الحياة للإنسان، والاعتبـار ات الاقتصـاديـة. فنحن نعيش في عالمين أحدهمـا هو العـالم الطبيعي من النبات، الحيو ان، التربة، الهو اء، الماء الذي سبقنا بملايين السنين و الذي نحن جزءًا منهه. العـالم الآخر هو العـادات والتقاليد الاجتمـاعبـة، وجميع الاختر اعات التي أوجدناها بأنفسنا باستخدام العلم و التكنولوجيا

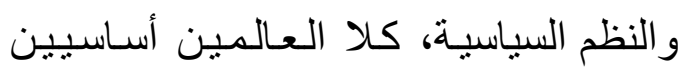

\section{خليل، محمد أحمد السبد}

قواعد علم البيئة / محمد أحمد السيد الهيد

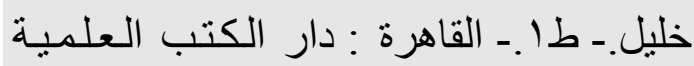

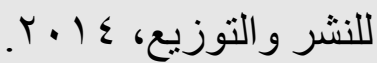

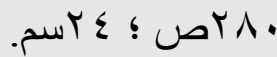
تدمك

خلال الخمسين عامًا المـاضيـة تغيرت العلاقة كثيرًا بين الإنسان و البيئة ؛ فقد بدأ ظهور حالات غريبـة على كوكب الأرض الأن وقد أصبحت الحضارة الحديثة و البيئة وجهًا لوجه لكل منهما. بالنسبة للبعض الاقتصـاد و التنمية الاجتماعية تعنى ببسـاطـة تدمير

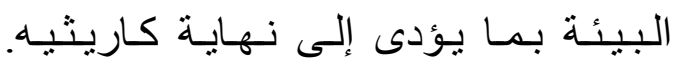
والانتقادات اعتبرت هؤلاء ضد التقدم والتكنولوجيا. هذا الخـلاف أدى إلى مفهوم جديد سمى البيئيون خلال الثمـانينيات من هن القرن الماضي. وظهر تحول كبير تجـاه حل المشاكل البيئية للـهو اء، و المـاء، و التـربـة،

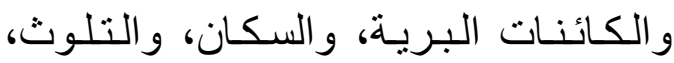
و الدفء الكوكبي، ونقص الأوزون، وفقد التنوع الحيوي. وكان الجهد على المستوى الدولي 


$$
\text { وماز الت مستمرة في تغير ها. }
$$

فالحياة بدأت على الأرض منذ مـا يزيـ عن ثلاثة بلايين من السنين. فى ذلك الوقت كان الغلاف الجوى يحتوى على النبتروجين، الأمونيا، الهيدروجين، أول أكسيد الكربون، الكوبل الميثان، بخار الماء ولكن لا يوجد الأكسجين الحر. مكونـات الغلاف الجوى هذه كانت بسبب الغاز ات الصاعدة من البر اكين النشطة ونظرًا لعدم وجود الأكسجين فلا توجد طبقة

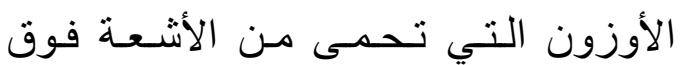
البنفسية التي تخترق بسهوله سطح الأرض الهرول

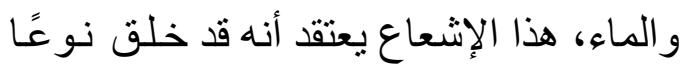
من التطور الكيميائي الذي أدى إلى تكوين الجزيئات العضوية مثل الأحماض ألأمينيـه التي أصبحت مكونات البناء للحياة الأولية.

\section{التطور النوعى والبيئة :} and environment

الأنواع في تصنيف الكائنات الحيـة هي وحدة بيولوجيه نتقاسم مجمو عـه جينات ور اثيه مشتركة ؛ فالتطور النوعي متبـاين الموقع أفترض أنه الآلية الأولى التي ظهرت

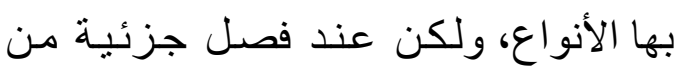
التجمعات الحرة المهجنـة بواسطة حواجز جغر افيه مثل الجزر أو الجبال لفترة زمنيبة كافية فإنها تفشل في أن ثُهَجَن حتى في حالة جمعهم معًا ؛ لذللك فإنها تتعـايش كأنواع متباينة فى عشش مختلفة، ولكن العزل
لحياتنا ولكن تكاملهما و اندماجهما معًا يتطلب تحمل الجهد.

\section{Environment : البيئة}

يمكن تعريفها بأنها الظروف والحـالات التي تحيط بالكائن الحي أو مجمـوعـة مـن الكائنـات الحيـة. والظروف الاجتمـاعيـه

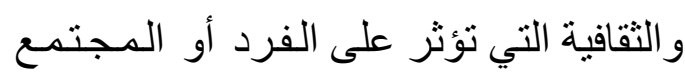
حيث إن البشر يعيش العالم الطبيعي والـعالم

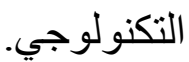

علم البيئة : هو الدراسة المنظمة لبيئنتـا ومكاننا فيها وهو يجمع علم البيولوجي ،

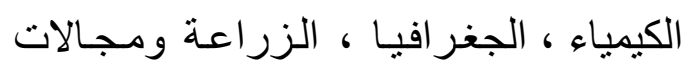
أخرى كثيرة الاستخدام وتلك المعلومـات لتحسين طريقه التعامل مع عالمنا. فعلم البيئة يتوجه طبقًا للمهمة و هو يتضمن انتا جميعًا

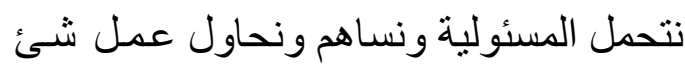

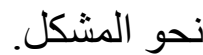

\section{Ecology and evolution : علم البيئة}

كوكب الأرض من كائناته الحية و البيئة (الهواء، الأرض، الماء ) التي تساند الحبـاة وتدعمها يعرف بـالغلاف الحيوي. كوكب

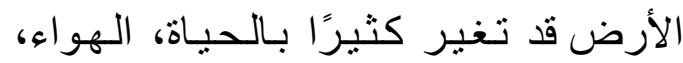
المحيطات، التربة، الصخور ، وتختلف البيئة كثيرًا عما تتكون عليه إذا كانت لكوكب بدون حياة، والحياة قد غيرت كثيرَا من سطح الأرض خلال الثناث ملايين سنـة المـاضيـة 
العو امل أو المملكات ذات سو 1 مقاطعة أو

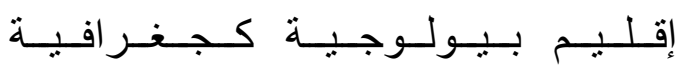
Biogeographically كل مقاطعة بيولوجية جغر افية تتكون من نظم بيـيـة (Ecosystems)، تكون

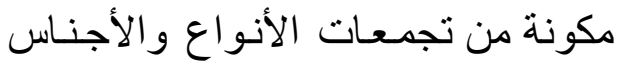
الحية الموجودة في منطقه بيئية، ويمكن

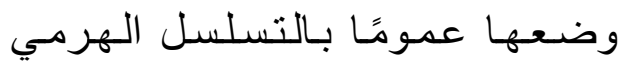
للمستويات ذات التنظيم البيولوجي وهذه بها المستويات الثناثة هي : • النظـام الإحيـائي أو التنـوع النظـامي الإحبائي • التوع أو تنوع الأجناس. • الصفـات الوراثيـة أو التنـوع الجيني الوراثي.

مبادئ الإيكولوجى : المادة ، الطاقة والحياة

\section{Principles of ecology:}

\section{$\underline{\text { matter energy and life }}$}

إن مجال الإيكولوجي هو لمـاذا وكيف

بتم تدوير المواد بين الأجزاء الحيـة وغير هوري حيه في بيئتنا، وكذلك الدر اسة العلميبة بين الكائنات الحية وبيئتها. علم الكائنـات الحية يـنطي مــالاً كبيـراً مـن (Biology) المو اضيع و المعايير من الجزيئات إلى النظم البيئية (Ecosystems) إلى النظم الكوكيبة. الإيكولوجي يختبر تواريخ الحياة، التوزيـع،
الجـر افي التـام ليس ضـروريًَا لتطور الأنواع، و أي الأنواع متجـانسـة الموطن أو النشأة الجغر افيـة قد تكون أكثر انتشـارًا

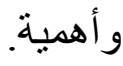

Genetics resources الموارد الوراثية المجموعات الطبيعيـة لمختلف أنواع النبات و الحيو انات (نبات وحيو انات، أو حقبة معينة) في مكان معين تشكل البيئة الحيـاتيـة للنبات و الحيوان فهي تبين الاختحلافات في مي مئي نوع ووفرة مختلف الأجنـاس. هذا التنوع الكثيف يسمى علميًا الحوض الوراثي وهو

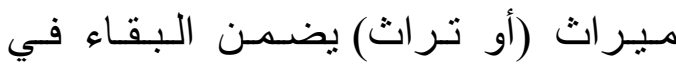
المستقبل. فعمليه تتوع الأجنـاس بدأت بعد وجود الحياة على كوكب الأرض وهي عملية متدرجة متأثرة بمختلف العو امل المناخيـة و الجغر افية والطبيعية منتجة سـلالات جديدة وأجنـاس دنيا.الأجنـاس التي لا تستطيع المواعمة مـع التخيرات المناخيـة تختفي

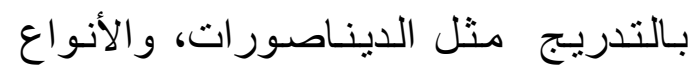
الأخرى من الثذييات الضخمة و الطيور التي كانت تحكم المملكة الحيو انيـة. الإنسـان عند القمة يمثل أفضل إنتاج للتحول وهو لا لإهل يختلف بأي حال ويزيد الأهمية عن الأنواع

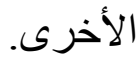

\section{Biodiversity : التّوع الحيوي}

يوجد على الأرض في ثمـانيـة من 
العلاقات الإنسانية مع المجال أو الغلاف الحيوي؟ وهو يشير إلى أنـا نستخدم كثيرًا من الموارد لإنتاج كثير من لن

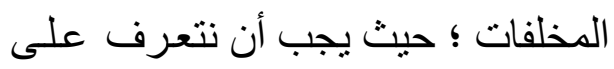
مصبر تلك المخلفات المنتجـة وأين تذهب? أين تذهب القمامة المنزلية؟ أيـن تـهـب الـــازات الـــادمـة مـن

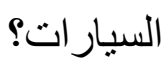
ما هي الطاقة :

الطاقة و المادة هما المكونـات الأسـاسيـة لكل من الكون والكائنـات الحيـة. إذا كانت المادة هي مادة الصنع للمنتجـات، الطاقة هي القدرة على عمل الثخل منثل تحريك المادة لمسافة. الطاقة يمكن أن تأخذ كثيرًا من الأشكال المختلفة لهنة حر ارة، ضوء، كهرباء، طاقة كيميائيـة تللك هي الأشكال العادية.

الطاقة الموجودة في أغر اض متحركة : تســى kinetic Energy الإليكترونيات التي تدور حول نواة الذرة - المياه التي تتدفق فوق الســالصخرة التي تنزلق من الجبل ـ الريـح الذي يهب خلال الأشجار.

\section{Potential Energy طاقه الوضع}

هي الطاقة المخزنة أي الكامنة ولكنهـا
وسلوك الأجناس، وكذلك بناء ووظيفة النظم الطبيعية على مستوى السكان، المجتمـعات، النظم البيئية، وشكل الأرض. نظم التعـامل مع الإيكولوجي نحو التفكير التاريخي حول التداخل الذي يجعل كل النظم أكثر من كونها مجرد مجموع أجز ائها الفرديـة. بمعنى أن كل كائن حي هو مصنسع كيمـاوي يمسك

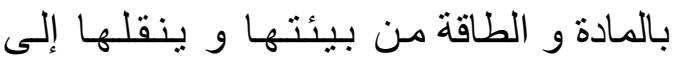
منشات و عمليات تجعل الحياة ممكنـة لذلك. لتفهم كيف تعمل النظم البيئية فإنه يكون من المهم أو لا معرفة شئ ما عن سـوك الط الطاقة و المادة في كل من الكائنات الحية وفى العالم.

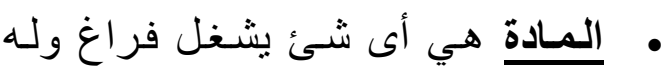
كتله.كل مادة لها ثلاث أشكال طبيعبية متغيرة بطريقه متداخلة أو مجـالات :

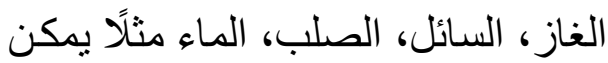
أن يوجد في شكل غـاز (بخـار المـاء) سـائل (مـاء) أو صـلب (ثلـج). في

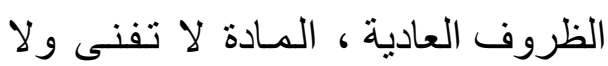
تستحدث ولكن يتم تدويرهـا مرات وررات. العناصر في جسم الإنسان، يتم تدوير ها خلال عدد من الكائنات الحيـة الأخرى خلال ملايين السنين. المادة يتم انتقالها و اتحادها بطرق مختلفة ولكنها لا لإن تختفي أي شـئ يذهب إلى أى مكان، ربما أن تلك المقولة هي المبدأ الطبيعي لني لبقاء المادة، كيف نطبق هذا المبدأ على 
كل الكائنات الحيـة تنكون من خـلايـا

الغرف الدقيقة التي تتم خـلالهـا عمليـات

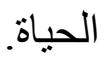

الكائنات المجهريه مثل البكتريا وبعض الطحالب، و البروتوز تتكون من خلايا واحدة بالمقارنة فإن جسم الإنسان بحتوي على عدد

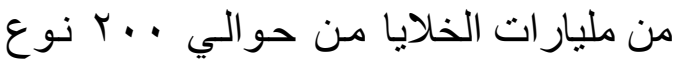
مميز يحيط بكل خلية غشـاء رقيق من الثحوم و البروبين الذي بستقبل المعلـومـات وينظم تدفق المواد بين الخلية وبيـتنها في الداخل، تنقسم الخلية إلى جزيئـات عضويـه داخل الخلية organelles، جسيمات الخلايـا الفر عية التي تمد الآلية للحياة. بعض من تلك جلك الجزيـئات العضـويـة داخل الخليـة تقوم بتخزين الطاقة و إطلاقها البعض الآخر تدير وتتظم وتوزع المعلومـات. البعض الآخر يكون البناء الداخلي الذي يعطى الخلية شكلها

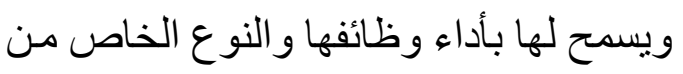
البروتينات - و الذي يسمى الأنزيمات - يعمل على تحفيز وتتشيط التفاعل الكيميائي بدون الاخول والاشتر الك في العملية. يوجد الآلاف من مختلف أنواع الأنزيمات المختلفة في كل خلية الكل مصمم للقبـام بعمليـات مختلفية تتوقف عليها الحياة. كيف يقتص التمثيل الضوئى للطاقة؟ How Does photosynthesis capture energy?
منتاحسة لـلاسـتخدام منثل الصـخرة الموجودة على قمة الجبل تحتوى على ملى طاقة وضع و التي تتحول إلى طاقـة حركية عند بدء تدحرج الصـخرة نـحو

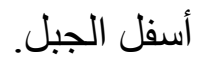
التفاعلات الكيميائية : تحدث عند كسر الأربطة و إعادة تكوينها بين الذرات و المركبات. تلك التفاعلات تكون المركبات الأساسية التي تعتمد

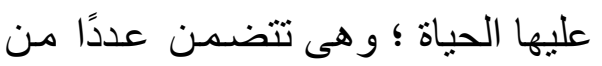
العمليات (و المشاكل) في علم البيئة. بعض التفاعلات مثل نكسر جزيئات السكر ، يمكن أن تكون شديدة التعقيد، ولكن كل التفاعلات تتبع المبادئ الأساسية للفيزياء : المـادة (الذرات) لا يمكن استحداثها أو فنائها، و الطاقة تنقل أو تنشتت مع حدوث التفاعل. هذا التفاعل الذي يحدث عندما تشعل مـوقد الغاز أو فرن. هذا التفاعل يبين كيف أن الذرات بعـاد تنظيمهـا إلى مركبـات

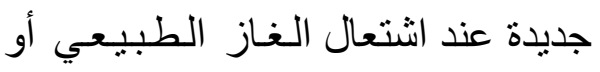
الميثنان، التفاعل يبدأ بو اسطة مدخلات

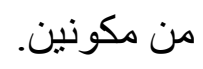
الخلايا : الوحدات الأساسية للحياة cell the fundamental units of life. 
المجتمع البيولوجي يتكون من كل

السكان التي تعيش وتتفاعل في منطقة معينة.

المجتمع البيولوجي وبيئته الطبيعيـ

(الماء) الموارد المعدنيـة ، الـهواء ، ضوء

الثمس ؛ ويشكل النظام الايكولوجي (البيئي) أو الكثير من الإيكولوجي يرتبط بتفهم طرق ولتُ

تحرك المادة والطاقة خلال النظم البيئية.

• الدورات البيولوجيـة والجيولوجيـة الـكـيـمـاويـة وعـمــليـات الـحـيـاة

\section{Biogeochmical cycles and life}

\section{processes}

العناصر و المركبات التي تؤازرنا تدور

بما لا نهاية خلال الأشياء الحية وخلال البيئة يثار إليه بالدوران البيولوجي الجيولوجي الكيماوي، المو اد يمكن أن تتحرك بسر عة أو بولي

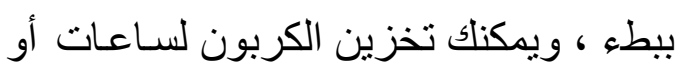
أيام بينما الكربون يتم تخزينـه في الأرض ملايين السنبين عندما يغير النشـاط البشري معدلات التدفق، أو أزمنة التخزين في تلك الدورات الطبيعية بما بربك قدرة البيئة على الى الئ التعامل معهم فإن تلك المو اد يمكن أن تصبح ملوثات الكبريت، النيتروجين، ثناني أكسيد الكربون، الفوسفور.

\section{الدورة المائية :}

مسار الماء خلال البيئة

معظم المبـاه الأرضيـة تخزن في
يحدث التمثيل الضوئي في الجزيئات

العضويـة داخل الخـليـة (organelles) الغشـائيـه التي تسـى البخضـور،الجزئ الوحيد الأخضـر الذي يقتنـص الطـاقـة الضـوئيـة ويستخدمهـا لعمل شـخل مفيد الكلوروفيل لا يقوم بهذه الوظيفة الهـامـة بمفرده ؛ حيث يوجد عديد من الجزيئات خلال أغشيه اليخضور تساهم في سلسلة من

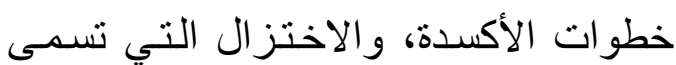
التفاعلات التي تعتمد على الضوء. الطاقة و الماع في البيئة :-

Energy and water in the environment

النظم الحية تستمـر بـاستخدام وتدوير

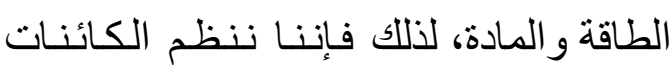
الحبـة بـالنسبـة للجنس ؛ وعدد السكان و المجتمعات و النظم البيئية والنوع أو الجنس غالبًا ما بعرف بأنة كل الكائنـات (kind) الحية التي ذات الصفات الور اثية (الجينيـة) المتشابهة. عدد السكان يتكون من كل عناصر النوع أو الجنس الذي يعيش في نفس المنطقة في نفس الوقت هنالك بعض الكائنات التي تكون من نفس النوع أو الجنس ولكن السكان تختلف حيث يعيشون في مناطق منفصلة جغر افيًا. 
بعد الظهر. أحيانًا التدوير (recycling) بستغرق وقتًا طويلًا الفحم و الزيت هما بقايـا

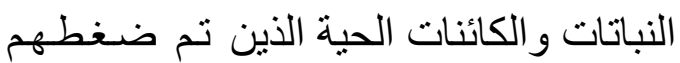
مـنـذ مـلايـيـن الســــن ذرات الكـربـون والهيدروجين، الأكسجين، النيتروجين، الكبريتـ الخ) لتللك الكائنات المدفونة لا يتم إطلاقها حتى حرق الزيت و الفحم، وكذلك توجد كميات ضخمة من الكربون محتجزة في شكل كربونات الكالسيوم المستخدم في بناء الهياكل و الصدفات للكائنات البحرية من الكائنات وحيدة الخلية. • دورة النيتروجين : النيتروجين يعتبر

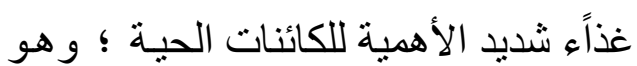
المكون الأول لكثير من الأسمدة الزر اعيـة

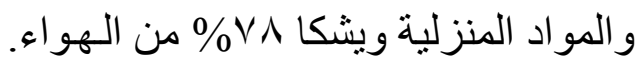
النبات يحتاج إلى النيتروجين خـلال دورة شديدة التعقيد، المفتـاح لـذهذ الدورة هو البكتريا. ويعود دخول النيتروجين إلى لهي البيئة بطرق عديدة.

• دورة الفوسفور : يعتبر الفوسفور مكون سمادي على مستوى الخلية فإن المركبات

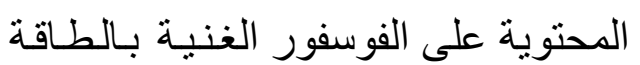
هي المساهم الأول في تفـاعلات انتقال الطاقة

كميه الفوسفور المتاحة في البيئة يمكنها أن تؤثر على الإنتاجيه بشكل مفاجئ، ووفرة الفوسفور يساعد على نمو الطحالب و النباتات
المحيطات ولكن الطـاقة الشـسيـة تبخر باستمر ار هذا المـاء والريـاح توزع بخـار الماء حول الكرة الأرضية.

المياه التي تتكثف فوق سطح الأرض في شكل المطر، الثلج أو الضبـاب. الكائنـات الحية تبعث البخـار الذي استخدمته خلال الشهيق و الزفير. هذه الرطوبة تعود و تدخل الجو أو تدخل البحير ات و المجاري المـائية ومنهم تعود إلى المحيط مع التحرك خلال لهل الكائنات الحية وخلال الجو فإن المـاء يكون مسئولًا عن عمليات الأيض خلال الخـلايـا لخديا لاستمرار تدفق الغذاء ولتوزيع الحرارة

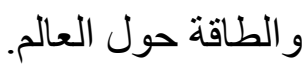
• دورة الكربون : فهو المكون البنائي للجزئيات العضوية ؛ حيث تبدأ مع التمثيل الضوئي للكائنات العضوية. مسار التدوير قد يكون سريعًا أو بطيًا. تصـور مـاذا بحدث لـجزئ السـكر الصغير الذي تبتلعه في زجاجة من عصير الفاكهة؟ جزئ السكر يمتص مجرى الدم حيث تكون متاحة للخلايا للتنفس الحلـوى لئري (للخلايا) أو لعمل جزئيات بيولوجيه أكثر more complex Biomelecules تعقيد إذا استخدم في التنفس فإنه يمكن زفير نفس ذرة الكربون في شكل بcor في سـاعـة أو اقل، والنبات يمكن أن يأخذ بcor الزفير هذي 
تسببت في استنز اف الموارد و إفساد المنـاخ الذي يهدد النظم البيئية التي نعتمد عليها في حياتنا تلك المخاوف عادة تؤدى إلى الحـاجـة إلى برامج ترشيد الإنجاب على مستوى العالم لخفض معدلات الخصوبـة وبـالتـالي استقرار أو حتى انكمـاش التعداد العـالمي ولي

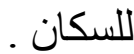

إحصائيات السكان وتقدير تغيرهم مع الزمن

\section{Human Demography}

علم الديموجر افي بشــل الإحصـائيـات

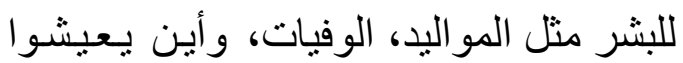
وكذلك إجمالي عدد السكان.

Food and الـغــذاء والـزراعـة

\section{-: Agriculture}

كلنا يحتاج إلى الطعام رغم أن التوقعات الرهيبة التي تنطلق بسر عـة نـحو الزيـادة السكانية سوف تؤدي عاجلاً إلى نقص الغذاء على المستوى العـالمي. إجمـالي إمدادات

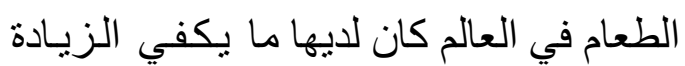
السكانية خلال القرنين الماضيين ولكن هل يمكن الاستمرار على هذا النحو مـع الزيـادة في السكان؟ وماذا يمكن أن يحدث فى البيئة إذا كان المطلوب منا هو توفير الطعام لـعدد

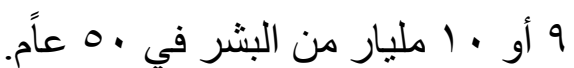
الجوع القارس والأمن الغذائي

\section{chronic Hunger and}

الخضر اء بمـا يجـعل الفوسفور المسـاهم الأساسي في تلوث المياه .

• دورة الكبريت : يلـعب دورًا هـامًا في الكائنات الحبـة خاصـة كمكون صغير ولكن أسـاسي للبروتينـات، مركبات الكبريت العـامل الحساسم الهام للمطر الحامضي وبالتالي على المباه السطحية، التربـة - الكبريت في شكل جسيمات صغيرة التي يحملهـا الهواء قد تعمل كمنظم هام للمناخ العالمي فدورة الكبريت معقدة بعدد كبير من حالات الأكسدة التي

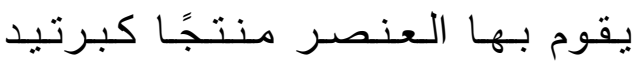
الهيدروجين وثاني أكسيد الكبريت و ايون الكبر بتاث.

\section{النمو السكانى population growth}

كل ثانية يولد أربع أو خمسة أطفال في أي مكان على كوكب الأرض في خلال نفس الثانية يموت اثنين آخرين من البشر. هذا الاختلاف بين الميلاد و الموت يعني الزيـادة في عدد السكان على مستوى العالم ْ.ب في الثانية هذا يعنى معدل النمو يقترب من

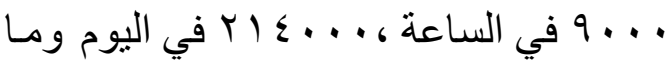
يقرب من AV مليون من البشر كل عـام. في عام 999 (م ز اد عدد سكان العالم عن 7 مليون ؛ وهذا جعلنا أكثر أنواع الفقاريـات على الأرض .الكثير من الناس ينزعج من هن الزيادة السكانية التي سوف تسبب أو ربما قد 
البيئة الصحية و السمومات

\section{Environmental health and}

\section{toxicology}

أنو اع المخاطر التي تتعرض لها الصحة و علاقتها بالبيئة وما هي الصحة الصّ؟ منظمـهـ الصحة العالمية (Who) تعرف الصحة بأنها حاله تمـام الرضـا الطبيعي والذهني لتهرفي و الاجتماعي وليس مجرد عدم وجود مرض ولرض

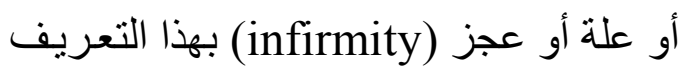
فإننا جميعًا مرضى إلى حد ما بالمثل، فإننا

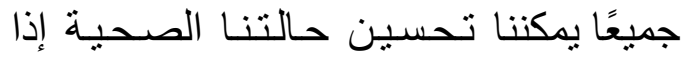
لنعيش سعداء ولعمر طويل ومنتج إذا كنا ننتبه إلى ما نفعله.

ما هو المرض؟ المرض هو التغبر

الضار في حالات الجسم بسبب عو امل بيئية التي يمكن أن تكون غذائية، كيماوية،

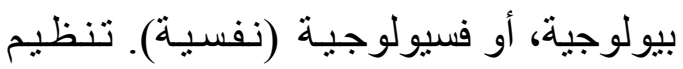
الغذاء والتغذية، عو امل العدوى، الكيماويات

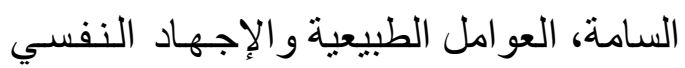
كل هذا له دور في المرض و الموت لمعرفـة الإنها كيف إن تللك العو امل تؤثر علينا دعنا نتعرف على بعض الأنواع الرئيسية ذات الخطورة على الصحة العامة. • الكيماويات والعوامل التي تسبب النمو غير طبيعي Teratogens بعض المركبات التي قد لا تكون ضـارة

\section{Food security}

إن إنتاج كميات كبيره من الطعام لا

يحقق توفير حاجة كل منهم .لقد قررت أننا

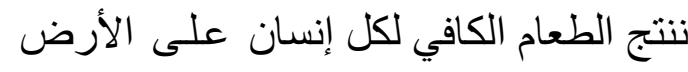

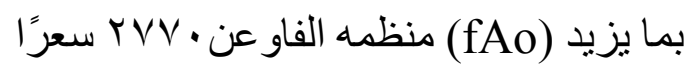

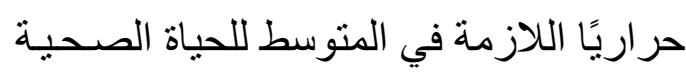
والنشطة، ولكن يقدر أن هنالك 10 مليون من البشر دون مستوى الغذاء المطلوب

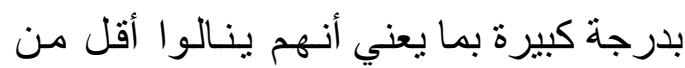

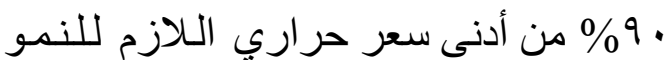
الطبيعي والتنمية و الحياة الصحية المنتجـة. ينطبق هذا الوصف على واحد من كل خمسة

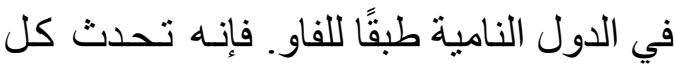

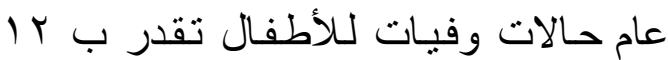
مليونًا بسبب سوء التغذية. • المصادر الرئيسية للغذاء major food -: resources من بين آلاف النباتات و الحيوانات

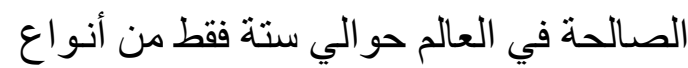

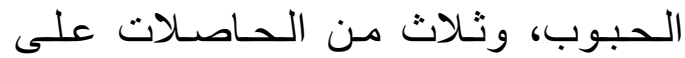
الجذريـة وحوالي · ب نوع من الفاكهة والخضروات وستة من الثدييات أثنين من نون الثناع الطبور المنزلية والقليل من الأسماك كن

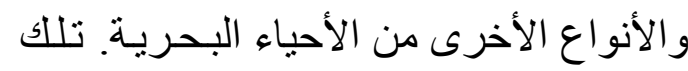
الأنواع هي التي توفر كل مصـادر الطعام الأنداء

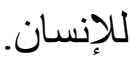




\section{The Atmosphere and}

\section{climate}

نحن نعيش عند قاع محيط من الهواء

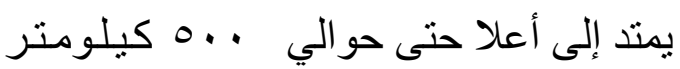

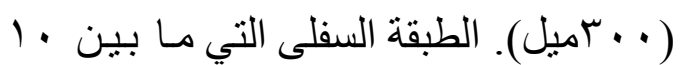

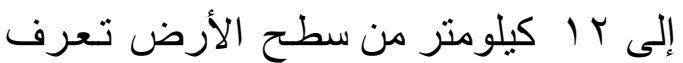
بطبقه التروبوسفير ؛ حيث يتحرك الهواء بدون انقطاع وينساب، ويتحرك كدوامات هو ائية مع استمرار إعادة توزيع الحرارة

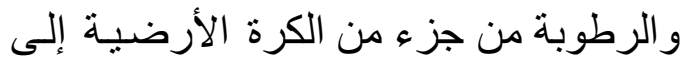
الآخر. مكونـات وسلوك التروبوسفير و الطبقات الأخرى التي تتحكم في المناخ (الحالات اليومية لدرجة الحرارة و الرطوبـة في الدكان)، وكذللك الإطار العام للمناخ على التى الته الددى الطويل. الهواء النظيف الجاف يكون معظمه من النيتروجين و الأكسجين. تركيز بخار الماء ينغير ما بين الصفر إلى •ـ؛ طبقًا لدرجة الحرارة والرطوبـة المتاحـة. الجسيمات الدقيقة ونقاط السائل و التي تسمي معًا الضباب تكون عالقة في الهو اء، وكذلك الضباب في الغلاف الجوي يقوم بدور هـام في نظام طاقة الأرض و إنتاج المطر.

\section{• تغير المناخ climate change}

ماذا يحدث في مناخ عالمنا؟ مـاذا يفعل الدب القطبي وإغراق الفقمة (عجل البحر
يمكن أن تسبب مشـاكل مؤلمـة في تللك المرحلة الحساسة من الحياة ربما يكون أكبر ماده مسببه لهذا الضرر هي الكحول. تناول الكحول خلال فترة الحمل يمكن أن يؤدي إلى هئ لهائ تداعيات خطيرة منها إعاقة النمو، ومشـاكل في السلوك وعيوب ذهنية، وعيوب في الجمجمة والذي يستمر طوال الحياة حتى أن شرب الكحول مرة واحدة في اليوم أنناء الحمل قد يصاحبه انخفاض في وزن المولود. • المسرطنات carcinogens :هي المواد المسببـة لمرض السرطان النمو الكبير في الخلية، وغير مسيطر عليه

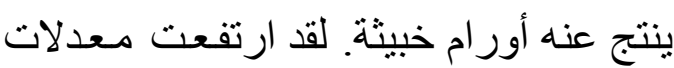
مرض السرطان فى الدول الصنـاعيـة خـلال القرن العشرين ؛ حيث أصبح مرض العرض العابه السرطان الآن من الأسباب الرئيسية لحسالات

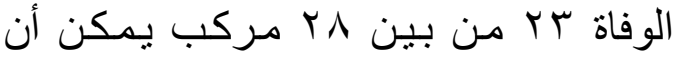
يكونو ا من المسرطنات، و السبب الكيمـاويات

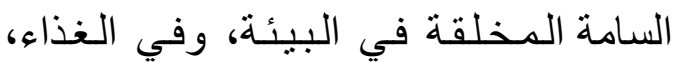
و البعض يعلل بسبب السلوك العام مثل : التنخين ، تناول الكحوليات، أنواع السرطان

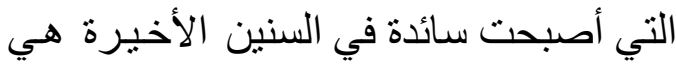
سرطان البروستاتا في الرجال وسرطان

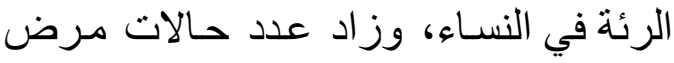
سرطان الثذي والجلد، ولكن حقق العلاج

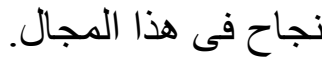
بيئة الهواء : الغلاف الجوى والطقس 
رغم أننا نظن ان الأرض أسفل أقدامنـا ثابتة ومستقرة ، إلا أن الأرض مليئة بـالقوة و النشاط ومتقلبة باستمر ار فالقوى الجبارة تـدور داخل الأرض مسبـبـة الانشطـار

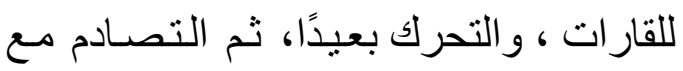
بعضها في تصادمات بطيئة وبدون فداحة.

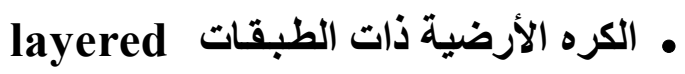
sphere :

القلب او داخل الأرض تتكون من كتلـة ساخنة كثيفة من المعدن غالبًا الحديد. تقطر آلاف الكيلومنرات صلب وجامد في المركز ولكن سائل فى خـارج القلب، تلك الكتلـة الضخمة تولا مجال مغناطيسي الذي بـلف

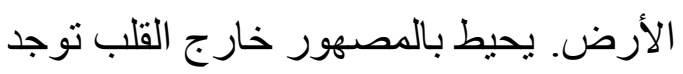

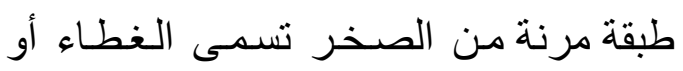
الحجاب وهو أقل كثافة عن القلب (اللب) ؛ حيث يحتوي على تركيز عالي من العنـاصـر الأخف مثل الأكسجين، السيليكون، المنجنيز. الطبقة الخارجية للأرض تكون باردة ، خفيفة الوزن، قثره صخريه هشة. القشرة أسفل المحيطات تكون كثيفة نسبيًا، خفيفة ، قديمـة حتى ^.ب مليون سنة ؛ حيث يتم بـاستمر ار إضافة ماده إضافية، وهي كذلك غـالبًا مـا تكون جر انيتية، بينما القشرة المحيطة تكون أساسًا من الصخر البازلت الكثيف.

توجد ثلاث تقسيمات رئيسية للصخور وهي : النـاريـة (Igneous)، التحويليـة (peps)، انتشار وبـاء الكوليرا في بيروو بأمريكا الجنوبية. انصهار ثلوج جبل كيلي وبلي منجـارو الثـهير، ندرة ميـاه الثـرب في الصين، الفيضانات غير عـاديـة الثديدة في بنجـلاديش، وبحر الثـواطئ فى لويسـانـا و اختفاء فراشة الألوان المختلفـة في جنوب كاليفورنيا؟ كل هذه الثـواهد تبدو أنها علامات التغير الكوكبي للمناخ والتي سوف تكون من أهم الموضو عات في علوم البيئة حاليًا. حدث تغيير للمناخ و على مقاييس زمنيـه كثيرة ومختلفة. البرودة المفاجئة منذ 10

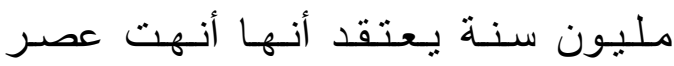

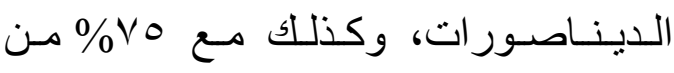
الكائنات الموجودة في ذلك الوقت. على المستوى الزمني الأقل فإن العصور الجليديـة العديدة التي استمر كل منها مئات الآلاف من السنين قد انتهت في المليونين سنة المساضيـة. حتى أن تحركات المناخ القصيرة قد حدثت مثل "عصر التلج القليل" الذي بدء في عام . . با وتسبب في تلف المحاصيل في كل أوروبا، على مستوى العقود الزمنيـة فإنها يبدو وجود دورات جفاف كل · r ع عامًا في أمريكا الثمالية. • بيئة طبقات الأرض والموارد الأرضية :Environmental geology and Earth resources:- 
الطاقة الفولتية الضوئية مhotovoltag

\section{Energy}

الخلية الفوتوفوليتية توفر جهد مثير نحو

اقتناص الطاقة الثمسية بالطريقة التي توفر

بها طاقه نظيفة متجددة متعددة الاستخدامـات.

هذه التجهيزة البسيطة ليس بـها أجزاء

متحركة، وتكاليف الصيانة تكاد تكون مهملة ولا تنجح أي ملوثات ولها عمر افتر اضي

يعادل ذلك لمحطة الطاقة بالوقود الحفري أو

بالطاقة النووية.

\section{• الطاقة المائية Hydropower •}

استخدم سقوط المياه المصدر للطاقة منذ

زمن بعيد. لقد كان اختر اع التربينات المـائية في القرن التاسع عشر السبب في زيادة كفاءة سدود الطاقة المائية في عام 9 ب ام أصبح

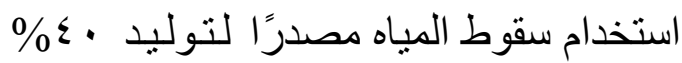
من الطاقة الكهربائية على مستوى العـالم عندئذ فإن طاقة إنتاج الكهربـاء بـالطـاقة المائية قد زاد بمقدار ما ضـعف ، ولكن استخدام الوقود الحفري قد زاد سريعًا بحيث

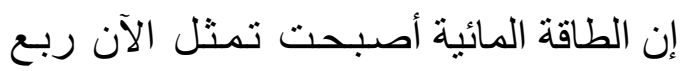
إجمالي الإنتاج من الطاقة الكهربية.

ماز ال كثير من دول العالم ينتج معظم

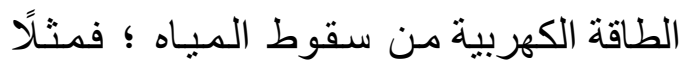

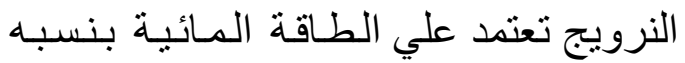

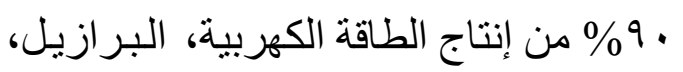

، (Metamorphic) (Sedimentary) • الطاقة النووية: الـ

في عام 1900 أعلن الرئيس الأمريكي

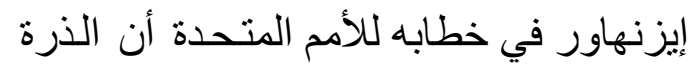
لأجل السلام كما أعلن أن الولايـات المتحدة فيدة سوف تبني مولدات الطاقة الكهربائية بالطاقة النووية لتوفير طاقه رخيصة ومتاحة.

توقع أن الطاقة النوويـة سوف تمـلأ العجز المتوقع في زيت البترول والغناز الطبيعي، ولكن مـع زيـادة تكاليف الإنشـاء و انخفاض الطلب على الطاقة الكهربائية ولية جعلت الطاقة النووية أقل جاذبية وخاصة من الن الن النها جانب الأمان الإشعاعي وان كانت الطـاقة النوويـة غير مسببـة لإنبعـاثـات غـاز الات وندات الاحتباس الحراري.

\section{• الطاقة الشمسية Solar Energy إن}

الثمس هي الفرن النووي الضخم في الفراغ ؛ حيث تمد باستمر ار كوكبنا بالطاقة. الحرارة الثمسيـة تحدث الريـاح، والدورة الهيدرولوجية (المائية) كل الكتلـة الحيويـة، وكذلك الوقود الحفري بـأنو اعـه متوسط ، كمية الطاقة الثمسية التي تصل أعلا الغلاف الجوي هي سب. إ وات متر مربع حوالي نصف هذه الطاقة يمتص أو ينعكس بواسطة

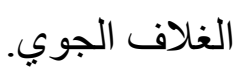


بنيوزيلندا، سويسر ا تلك الدول الثالاث تنتج ما لا يقل عن 0٪\% من الكهربـاء بـاستخدام الطاقة المائية. • طاقه الرياح wind Energy .

تمت تسمية الهواء حول الأرض بأنـه بطارية تخزين طاقه شمسيه بحجم ، r مليار

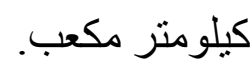

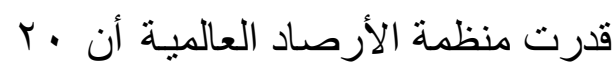
مليون ميجا وات من طاقه الريـاح يمكن الحصول عليها تجاريًا في جميع أنحاء العالم وليس من بينها المساهمات من مـمـوعات طواحين الهواء عند البحر وهذا يعـادل مهل مهول حو الي • م ضعف الطاقة الحالية المنتجه من فن فئن المحطات النووية في كل أنحاء العالم. • يفيد القارئ المتخصص في شئون البيأسة والمكتبة المتخصصة فهو يعمل على حل المشاكل البيئية للهواء والمساء والتربـة،

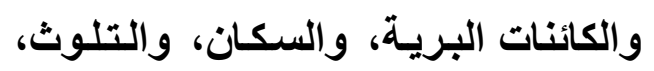
والدفء الكوكبي، نقص الأوزون، وفقد التنوع الحيوي. وقد تحقق نجاحات كثيرة

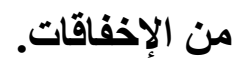

السبب في تلكك الإخفاقـات هو نقص

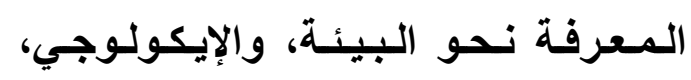
والوظائف الطبيعية. ولقد ثبت أن اللجوع الي تعليم البيئة هو العلاج. 